\title{
Accurate Estimation of Primary User Traffic Based on Periodic Spectrum Sensing
}

\author{
Ahmed Al-Tahmeesschi* ${ }^{*}$ Miguel López-Benítez*, Janne Lehtomäki ${ }^{\dagger}$ and Kenta Umebayashi ${ }^{\ddagger}$ \\ ${ }^{*}$ Dept. of Electrical Engineering and Electronics, University of Liverpool, United Kingdom \\ $\dagger$ Centre for Wireless Communications, University of Oulu, Finland \\ $\ddagger$ Graduate School of Engineering, Tokyo University of Agriculture and Technology, Japan
}

\begin{abstract}
An accurate estimation of the primary statistics is essential for Cognitive Radio (CR) systems. This knowledge can be exploited to enhance $C R$ performance and reduce the interference with the primary users. In this work, we propose a method based on the Method of Moments (MoM) to improve the distribution estimation. A Modified Method of Moments (MMoM) with a correction factor is proposed to improve the estimation of moments and thus the resulting primary distribution. The simulation and experimental results show that the MMoM approach is notably more accurate. Finally, we study the importance of having a sufficiently large sample space and the effect of sample size on the moments and the primary distribution estimation.
\end{abstract}

Keywords- Cognitive radio, spectrum sensing, primary activity statistics

\section{INTRODUCTION}

Cognitive Radios (CR) are proposed as a solution for the spectrum scarcity problem by allowing secondary users (SU) to access primary users (PU) channel (spectrum holes) in an opportunistic and non-interfering manner. Spectrum sensing is essential to reduce the interference $[1,2]$.

CR have to work in a fast and accurate manner to identify empty slots in the spectrum. In order to improve the performance of $\mathrm{CR}$, the knowledge of previous spectrum occupancy pattern (distribution) can be exploited to improve the system performance [3]. Statistical information such as the duration of past busy/idle periods, duty-cycle, their minimum, mean and variance, or the underlying distribution can be used to access the spectrum more effectively by selecting the most appropriate channel for transmission [4] and enhancing the forecasting of PU occupancy pattern to minimize the interference [5].

The activity statistics of the primary channels are initially unknown to the CR users and are estimated using spectrum sensing decisions. CR users sense the channel state periodically and in every sensing event a binary decision (idle/busy) is made based on an appropriate spectrum sensing (signal detection) algorithm [6]. While the main purpose of spectrum sensing is the detection of transmission opportunities [7], the sequence of spectrum sensing decisions can also be used to estimate the durations of the idle and busy periods and their statistics. In this case the spectrum sensing function is divided into two layers: the first layer is in charge of detecting the instantaneous on/off state and the second layer is in charge of spectrum awareness and the estimation of PU activity [8].

The estimation of primary activity statistics by means of periodic channel observations is impaired by several practical limitations that determine the accuracy to which such statistics can be known by the CR system. Firstly and most importantly, the use of a finite sensing period imposes a fundamental limit on the temporal resolution to which the duration of idle and busy periods can be measured. Secondly, channel statistics need to be inferred based on a limited number of channel observations (samples). The objective of this work is to improve the estimation of PU activity statistics (focusing on the distribution of PU activity busy/idle periods). A novel method is proposed based on the Method of Moments (MoM) to improve the PU distribution estimation under spectrum sensing impairments. The impact of sensing errors (i.e., false alarms and missed detections) is out of the scope of this work and hence a high signal-to-noise ratio (SNR) scenario with no sensing errors is considered. The low SNR scenario with spectrum sensing errors will be considered in future work.

The contribution of this work is threefold:

1) A novel method to palliate the effects of spectrum sensing on the distribution estimation is proposed.

2) The effects of sample size on the estimation of moments at the SU side is studied.

3) Experimental results are provided to validate the proposed method and simulations.

The remainder of this paper is organized as follows. First, Section II describes the system model considered in this work. The effect of sensing period on the moments estimation is analysed in Section III. Section IV presents the proposed distribution estimation methods. The simulation and experimental results of the proposed methods are analysed thoroughly in Section V. Finally, Section VI concludes the paper.

\section{System Model}

In this paper, a single $\mathrm{SU}$ is considered to detect PU activity. The SU performs spectrum sensing decisions with periodicity $T_{s}$ time units (t.u.) to detect the presence/absence of PU signal on a specific frequency band. The results of the decisions are introduced as a binary alternating state: busy when the PU signal is present at the SU and idle when the PU signal is absent at the SU. The computed elapsed time (at SU) between two PU state changes is considered as an estimation $\widehat{T}_{i}$ of the real period duration $T_{i}(i=0$ for idle periods and $i=1$ for busy periods) as illustrated in Fig. 1, where the estimation of the duration of a busy is shown (idle periods can be estimated using the same method). The estimated period durations are integer multiples of $T_{s}$ (i.e., $\left\{\widehat{T}_{i, n}\right\}_{n=1}^{N_{s}}=k T_{s}$, with $k, n, N_{s} \in N^{+}$, where $k$ represents the number of sensing 


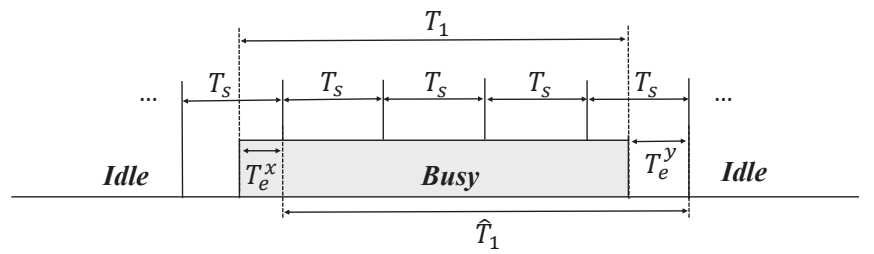

Fig. 1. Considered model. $T_{s}, T_{1}, \widehat{T}_{1}$ represent the sensing period, original busy period and estimated busy period, respectively. $T_{e}^{x}$ and $T_{e}^{y}$ are the errors in period estimation.

events within the estimated period, $n$ represents the period index such that more recent periods have higher index values and $N_{s}$ is the number of transmitted periods). A similar model was considered in $[9,10]$.

Primary activity statistics can be estimated based on the observed idle/busy period durations. To this end, CR users sense the channel with a specific periodicity duration of $T_{s}$ (assumed to be lower than or equal to the minimum PU activity duration). Based on these channel states observations at specific sensing instants, CR makes an estimation $\widehat{T}_{i}$ of the real period duration $T_{i}$, which can be modelled as $\widehat{T}_{i}=T_{i}+T_{e}$, where $T_{e}$ represents the error component in the estimation of $T_{i}$ as a result of spectrum sensing. $T_{e}$ is represented by the sum of two error components (i.e., $T_{e}=T_{e}^{y}-T_{e}^{x}$ ), where both of $T_{e}^{x}$ and $T_{e}^{y}$ can take values within the interval $\left[0, T_{s}\right]$. Hence they can be assumed to be uniformly distributed random variables (i.e., $T_{e}^{x}$ and $T_{e}^{y} \sim U\left(0, T_{s}\right)$ ) independent of each other (a similar assumption can be found in $[10,11])$. Since $T_{e}^{x}$ and $T_{e}^{y}$ are assumed independent of each other, then the pdf of the combined error $T_{e}$ (which is the sum of $T_{e}^{y}$ and $\left.-T_{e}^{x}\right)$ can be obtained as the convolution of the distributions of $T_{e}^{y} \sim U\left(0, T_{s}\right)$ and $-T_{e}^{x} \sim U\left(-T_{s}, 0\right)$ [12], which leads to a symmetric triangular distribution with a width of $2 T_{s}$.

To account for the discrete feature of the estimated periods, a second model is here considered. It can be shown that the estimated periods can also be expressed as:

$$
\widehat{T}_{i}=\left(\left\lfloor\frac{T_{i}}{T_{s}}\right\rfloor+\xi\right) T_{s}
$$

where $\lfloor\cdot\rfloor$ denotes the floor operator and $\xi \in\{0,1\}$ is a Bernoulli random variable introduced to reflect the fact that the same original period $T_{i}$ can lead to two possible estimated periods, either $\widehat{T}_{i}=k T_{s}$ or $\widehat{T}_{i}=(k+1) T_{s}$, depending on the relative (random) position of the sensing events with respect to the beginning/end of $T_{i}$.

\section{iII. Estimation of the Minimum, Mean, Variance AND DUTY CYCLE}

\section{A. Estimation of the Minimum Period Duration}

As mentioned earlier, CR systems estimate the PU activity pattern based on the discrete observation periods $\widehat{\tau}_{i}=$ $\left\{\widehat{T}_{i, n}\right\}_{n=1}^{N_{s}}$, where $N_{s}$ represents the number of observed periods (sample size of $\widehat{\tau}_{i}$ ). In order to have accurate estimations of the distribution, the sample size $N_{s}$ has to be sufficiently large. The minimum activity duration was simulated in [9], nevertheless we provide a closed form expression for the estimation of minimum PU activity time $\left(\widehat{\mu}_{i}\right)$ and link it to the sensing period $\left(T_{s}\right)$. The proposed expression is based on the second probabilistic model described in Section II:

$$
\begin{aligned}
\widehat{\mu}_{i}=\min \left(\widehat{\tau}_{i}\right) \approx \min \left(\widehat{T}_{i}\right) & =\min \left\lceil\left(\left\lfloor\frac{T_{i}}{T_{s}}\right\rfloor+\xi\right) T_{s}\right\rfloor \\
& =\left\lfloor\frac{\mu_{i}}{T_{s}}\right\rfloor T_{s}
\end{aligned}
$$

Note that the minimum value in (2) corresponds to $\min \left(T_{i}\right)=$ $\mu_{i}$ and $\min (\xi)=0$.

\section{B. Estimation of the Mean and Variance of Period Durations}

Given a set of $N_{s}$ discrete periods $\widehat{\tau}_{i}=\left\{\widehat{T}_{i, n}\right\}_{n=1}^{N_{s}}$, the mean $\mathbb{E}\left(\widehat{T}_{i}\right)$ and variance $\mathbb{V}\left(\widehat{T}_{i}\right)$ of the provided durations can be estimated based on the corresponding sample moments:

$$
\begin{gathered}
\mathbb{E}\left(\widehat{T}_{i}\right)=\frac{1}{N_{s}} \sum_{n=1}^{N_{s}} \widehat{T}_{i, n} \\
\mathbb{V}\left(\widehat{T}_{i}\right)=\frac{1}{N_{s}-1} \sum_{n=1}^{N_{s}}\left(\widehat{T}_{i, n}-\mathbb{E}\left(\widehat{T}_{i}\right)\right)^{2}
\end{gathered}
$$

The impact of $T_{s}$ on the estimated moments (first and second) can be determined as follows:

$$
\begin{gathered}
\mathbb{E}\left(\widehat{T}_{i}\right)=\mathbb{E}\left(T_{i}\right)+\mathbb{E}\left(T_{e}\right)=\mathbb{E}\left(T_{i}\right) \\
\mathbb{V}\left(\widehat{T}_{i}\right)=\mathbb{V}\left(T_{i}\right)+\mathbb{V}\left(T_{e}\right)=\mathbb{V}\left(T_{i}\right)+\frac{T_{s}^{2}}{6}
\end{gathered}
$$

where $T_{i}$ and $T_{e}$ are assumed to be independent of each other, and $\mathbb{E}\left(\widehat{T}_{e}\right)$ and $\mathbb{V}\left(\widehat{T}_{e}\right)$ have been replaced with the mean and variance of the triangular distribution described in Section II. The triangular distribution in this case has a mean value of zero (i.e., $\mathbb{E}\left(T_{e}\right)=0$ ), which means that the duration of $T_{s}$ does not affect the calculation of the mean value. On the other hand, the calculation of the variance is affected by a factor of $T_{s}^{2} / 6$. Based on (6), the effect of $T_{s}$ can be minimized by applying to (4) the appropriate correction factor:

$$
\mathbb{V}\left(\widetilde{T}_{i}\right)=\mathbb{V}\left(\widehat{T}_{i}\right)-\frac{T_{s}^{2}}{6}
$$

where $\mathbb{V}\left(\widetilde{T}_{i}\right)$ is the observed variance after correction. This approach eliminates the impairments imposed by sensing operation with duration of $T_{s}$ in the estimated moments and is able to provide an accurate estimation of the real moments of $T_{i}$, based on the estimated period durations $\widehat{\tau}_{i}$, as long as the sample size $N_{s}$ is sufficiently large.

\section{Estimation of the Duty Cycle}

One of the important statistics of PU activity to represent the channel usage is the average duty cycle $(\Psi)$. Assuming a large sample size is observed, then the average duty cycle can be estimated as:

$$
\widehat{\Psi}=\frac{\mathbb{E}\left(\widehat{T}_{1}\right)}{\mathbb{E}\left(\widehat{T}_{0}\right)+\mathbb{E}\left(\widehat{T}_{1}\right)}
$$

As the value of the mean of the estimated periods is unchanged as explained in Section III.B, then the estimated $\widehat{\Psi}$ will be the same as the real one (i.e., $\widehat{\Psi} \approx \Psi$ ). It is worth noting that $\widehat{\Psi}$ is not affected by the sensing period $T_{s}$. 


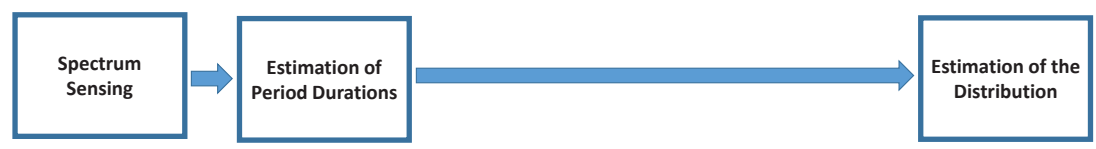

(a)

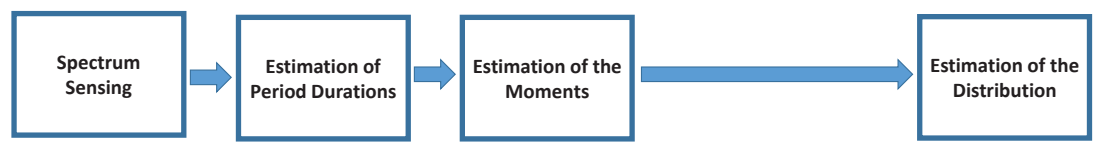

(b)

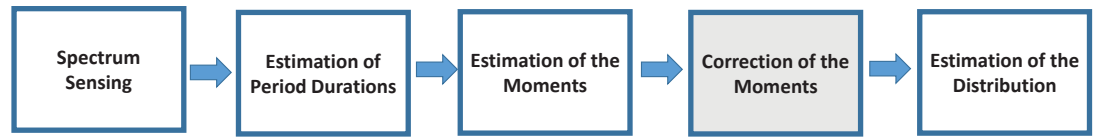

(c)

Fig. 2. Distribution estimation methods : (a) Direct estimation, (b) Method of Moments (MoM), (c) Modified Metod of Moments MMoM.

\section{Estimation OF THE Distribution}

Three methods are considered to estimate the distribution.

\section{A. Direct Estimation}

The direct estimation method is based on the calculation of the empirical cumulative distribution function (ecdf function in MATLAB). The ecdf function calculates the Kaplan-Meier estimate of the provided samples [13]. The flowchart of this proposed strategy is illustrated in Fig. 2(a). The main drawback of this method is that the estimated distribution is discrete as the values of the estimated periods are integer multiples of the sensing period (i.e., $\widehat{T}_{i}=k T_{s}, k=1,2,3, \ldots$ ). Moreover, it is not possible to apply any correction factors to this estimation method, which affects its accuracy.

\section{B. Method of Moments (MoM) Estimation}

To overcome the first drawback of the direct estimation method, a solution based on MoM is proposed. Instead of estimating the distribution of the PU activity periods directly from the periods themselves, which produces a discrete distribution, this method computes first the moments (mean and variance of the PU activity periods) and then estimates the parameters of the distribution based on the MoM assuming a certain distribution model. The flowchart of this proposed strategy is illustrated in Fig. 2(b). The sample moments are equated to the distribution moments and then by solving the resulting equations the distribution parameters are obtained. As opposed to the previous method, the resulting distribution with this approach is continuous instead of discrete, thus offering the possibility to minimize the impact of sensing period.

Various methods have been proposed to estimate the distribution parameters besides MoM such as, Maximum likelihood Estimation (MLE) and Least Squares Estimation (LSE). In this work, we only consider MoM-based solutions. Even though other methods might provide a better distribution parameters fit, they require the complete history of past observed period durations while with MoM the distribution moments can be estimated from sample moments, which can be computed recursively based on last samples. As a result, the practical implementation of MoM-based solutions would result in significantly lower computation and memory cost for CR devices.

In this work, we assume the state holding times of PU $\left(T_{0}\right.$ and $\left.T_{1}\right)$ follow a Generalized Pareto (GP) distribution, which was proven to give best accuracy fit with a reasonable complexity in comparison with other more complex distributions [14]. The busy and idle durations are also assumed to be independent of each other [15]. The probability density function (pdf) and cumulative distribution function (cdf) for the GP distribution are given, respectively, as [16]:

$$
\begin{aligned}
& f_{T_{i}}(t)= \begin{cases}0 & t<\mu_{i} \\
\frac{1}{\lambda_{i}}\left[1+\frac{\alpha_{i}\left(t-\mu_{i}\right)}{\lambda_{i}}\right]^{-\left(1 / \alpha_{i}+1\right)} & t \geq \mu_{i}\end{cases} \\
& F_{T_{i}}(t)= \begin{cases}0 & t<\mu_{i} \\
1-\left[1+\frac{\alpha_{i}\left(t-\mu_{i}\right)}{\lambda_{i}}\right]^{-1 / \alpha_{i}} & t \geq \mu_{i}\end{cases}
\end{aligned}
$$

where $\alpha_{i}$ and $\lambda_{i}$ are the shape and scale of the GP distribution respectively, and $\mu_{i}$ is the location (also the minimum PU activity duration). Moreover, $T_{i} \geq \mu_{i}, \alpha_{i} \geq 0, \lambda_{i} \geq 0$. The mean and variance of the GP distribution are expressed as:

$$
\begin{gathered}
\mathbb{E}\left(T_{i}\right)=\mu_{i}+\frac{\lambda_{i}}{1-\alpha_{i}} \\
\mathbb{V}\left(T_{i}\right)=\frac{\lambda_{i}^{2}}{\left(1-\alpha_{i}\right)^{2}\left(1-2 \alpha_{i}\right)}
\end{gathered}
$$

The expressions needed to estimate the parameters of the GP distribution from the sample moments can be obtained by solving (11) and (12) for such parameters, which yields:

$$
\widehat{\mu}_{i}=\min \left(\widehat{T}_{i}\right)
$$




$$
\begin{gathered}
\widehat{\alpha}_{i}=\frac{1}{2}\left(1-\frac{\left(\mathbb{E}\left(\widehat{T}_{i}\right)-\widehat{\mu}_{i}\right)^{2}}{\mathbb{V}\left(\widehat{T}_{i}\right)}\right) \\
\widehat{\lambda}_{i}=\frac{1}{2}\left(1+\frac{\left(\mathbb{E}\left(\widehat{T}_{i}\right)-\widehat{\mu}_{i}\right)^{2}}{\mathbb{V}\left(\widehat{T}_{i}\right)}\right)\left(\mathbb{E}\left(\widehat{T}_{i}\right)-\widehat{\mu}_{i}\right)
\end{gathered}
$$

where $\widehat{\lambda}_{i}$ and $\widehat{\alpha}_{i}$ are the estimated values of original $\lambda_{i}$ and $\alpha_{i}$. Introducing the MoM estimates provided by (13), (14) and (15) into (9) and (10) provides a continuous estimation of the distribution of PU activity periods.

Notice that the location parameter $\mu_{i}$ can be estimated as shown in (13) since it corresponds to the minimum period duration. However, such estimation will be affected by the employed sensing period $T_{s}$. In many cases SU may be able to have a perfect knowledge of this parameter, for example in the case of primary systems that use some form of regional beacon signals with real-time information [17] or when the radio technology of the primary system is standardised and known (e.g., the slot duration of GSM).

\section{Modified Method of Moments (MMoM) Estimation}

The MoM solution discussed in the previous section solves the problem of the estimation error introduced by the discrete distribution resulting from the direct estimation method. However, as shown in the analysis of Section III, the estimated moments may have an error component resulting from the use of a finite sensing period $T_{s}$. This motivates the introduction of a Modified Method of Moments (MMoM) solution. The flowchart of this proposed strategy is illustrated in Fig. 2(c). The main difference with respect to the MoM method is the correction of the estimated moments, which is shaded in Fig. 2(c)

Based on (7), the new distribution parameters can be estimated as follows:

$$
\begin{gathered}
\widetilde{\alpha}_{i}=\frac{1}{2}\left(1-\frac{\left(\mathbb{E}\left(\widehat{T}_{i}\right)-\widehat{\mu}_{i}\right)^{2}}{\mathbb{V}\left(\widetilde{T}_{i}\right)}\right) \\
\widetilde{\lambda}_{i}=\frac{1}{2}\left(1+\frac{\left(\mathbb{E}\left(\widehat{T}_{i}\right)-\widehat{\mu}_{i}\right)^{2}}{\mathbb{V}\left(\widetilde{T}_{i}\right)}\right)\left(\mathbb{E}\left(\widehat{T}_{i}\right)-\widehat{\mu}_{i}\right)
\end{gathered}
$$

Notice that (16) and (17) are similar to their counterparts in (14) and (15), respectively, but are based on a corrected version of the moments. In particular, the corrected variance $\mathbb{V}\left(\widetilde{T}_{i}\right)$ is used instead of the sample variance $\mathbb{V}\left(\widehat{T}_{i}\right)$, while the sample mean does not need correction as inferred from (5).

\section{NUMERICAL AND EXPERIMENTAL RESULTS}

This study assumes that the sensing period is lower than or equal to the minimum PU activity time $\left(T_{s} \leq \mu_{i}\right)$. This is required to ensure that no PU activity periods are missed in the sensing process (the shortest detectable period is $T_{s}$ ), which would otherwise lead to significant estimation errors. Notice that this consideration implicitly assumes that the minimum activity time of the PU, $\mu_{i}$, is known to the $\mathrm{SU}$ so that the value of $T_{s}$ can be configured not to exceed $\mu_{i}$. This assumption is realistic as discussed above.

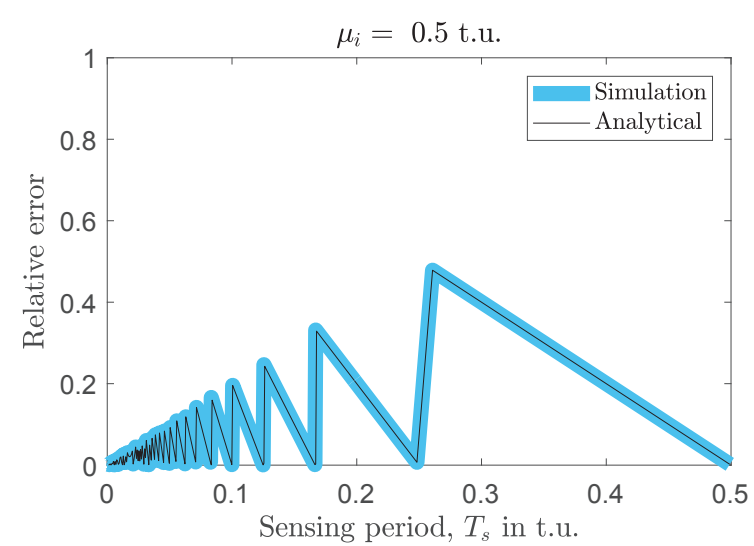

Fig. 3. Relative error of the estimated minimum period $\widehat{\mu}_{i}$.

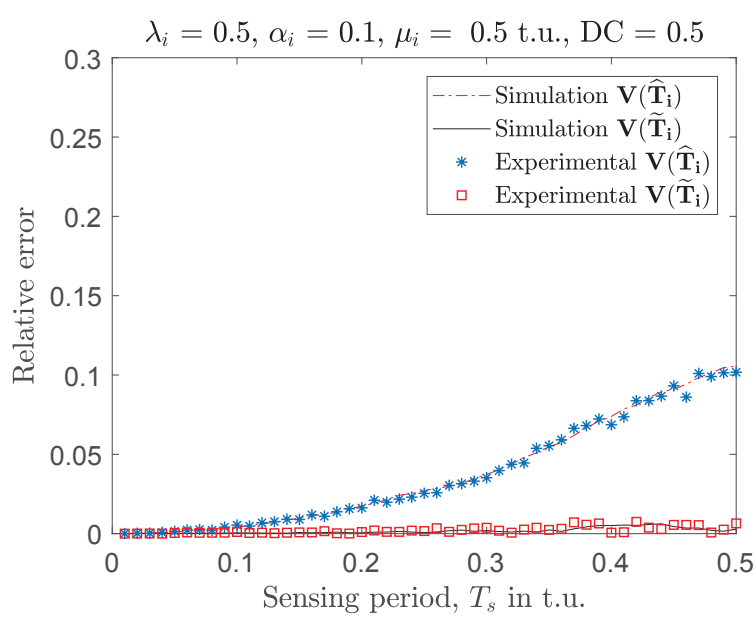

Fig. 4. Relative error of the calculated variance using (6) and (7).

The hardware experiments are conducted with a Prototype for the Estimation of Channel Activity Statistics (PECAS) [18]. The transmitter (primary user) sends a sequence of GP-distributed idle/busy periods utilising an ON-OFF Keying (OOK) modulator with an output power of $2 \mathrm{dBm}$ and the operating frequency is $433 \mathrm{MHz}$. The receiver (secondary user DSA/CR) uses a Software-Defined Radio (SDR) with a gain of $20 \mathrm{~dB}$ to monitor the transmitter activity (busy/idle) at 433 $\mathrm{MHz}$ every $T_{s}$ seconds. To ensure a high power reception the transmitter and receiver are separated by 1 metre distance. At every sensing event, signal samples are captured at a sample rate of $10^{6}$ samples per second, which are processed to decide the channel activity state using energy detection. The outcomes of the energy detection decisions are used to estimate the durations of the observed idle/busy periods and compute the primary activity statistics.

First, the effect of desynchronized spectrum sensing on the observed minimum period will be discussed. In order to quantify this effect, the relative error $\left(R_{e}\right)$ metric is utilized and calculated as $R_{e}=\left(\left|\widehat{\mu}_{i}-\mu_{i}\right|\right) / \mu_{i}$. Fig. 3 shows the relative error between the minimum sensed period $\widehat{\mu}_{i}$ versus the original minimum $\mu_{i}$ as a function of the sensing period. As it can be appreciated from Fig. 3, the analytical expression 


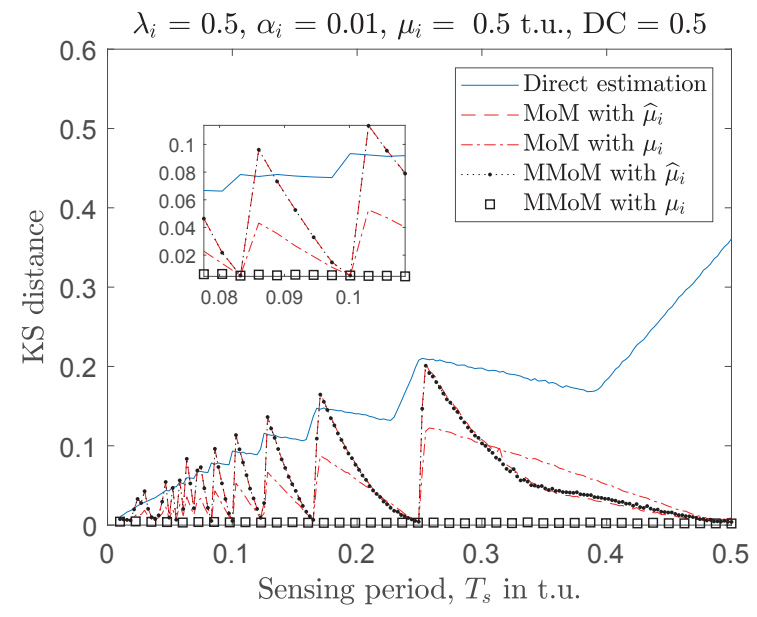

Fig. 5. The KS distance using direct estimation, MoM and MMoM.

provides a perfect agreement with the simulation results. The estimated value of $\widehat{\mu}_{i}$ depends on $T_{s}$ and the relative error shows an oscillating pattern with zeros at $T_{s}$ values that are integer sub-multiples of the original $\mu_{i}$.

Fig. 4 shows the relative error of the calculated variance with (4) and (7). As appreciated, the variance estimated using (4) is accurate only for low values of the sensing period $T_{s}$, while the estimation obtained using (7) is accurate regardless of the value of $T_{s}$, which proves that the correction factor in (7) can reduce significantly the estimation error of the variance. These results are verified experimentally using PECAS with a nearly perfect match with the simulation results.

To measure the similarity between the estimated and original distributions, the Kolmogorov-Smirnov (KS) distance [19] is utilized. The KS distance is defined as:

$$
D_{K S}=\sup _{t}\left|F_{T_{i}}(t)-F_{\widehat{T}_{i}}(t)\right|
$$

where $F_{T_{i}}(t)$ and $F_{\widehat{T}_{i}}(t)$ are the cdfs of the original and observed periods, respectively.

Fig. 5 shows the KS distance as a function of the sensing period for the considered distribution estimation methods. Two cases are shown for the MoM and MMoM methods, one where the value of the location parameter is estimated from spectrum sensing observations as indicated in (13) (labelled as 'with $\widehat{\mu}_{i}{ }^{\prime}$ ) and another where the true value of the location parameter is assumed to be known by the SU (labelled as 'with $\mu_{i}$ ').

First thing to notice in Fig. 5 is that the direct estimation method results in a significantly higher estimation error than the MoM and MMoM methods. In fact, the direct estimation method can provide an accurate estimation of the distribution of PU activity periods only if the employed sensing period is very short. The MoM and MMoM methods can provide in general more accurate estimations over the whole range of $T_{s}$ values. The results obtained for the MoM and MMoM methods indicate that the estimation error is zero when the employed sensing period $T_{s}$ is an integer sub-multiple of the true minimum PU activity time $\mu_{i}$. Notice that the same behaviour is observed for the relative error of the estimated minimum $\widehat{\mu}_{i}$ in Fig. 3, which suggests that an accurate estimation (or perfect knowledge) of the value of $\mu_{i}$ can improve significantly the accuracy of the estimated distribution. To corroborate this observation, the accuracy of the MoM and MMoM methods is shown in Fig. 5 for the cases where the minimum PU activity time is known by the SU ('with $\mu_{i}$ '), and when it is estimated as indicated in (13) and is therefore not perfectly accurate ('with $\widehat{\mu}_{i}^{\prime}$ '). As it can be observed, the distribution can be estimated more accurately when the minimum PU activity time $\mu_{i}$ is known accurately. However, for the MoM method this is not enough to provide a sufficient level of accuracy as seen in Fig. 5 since the effect of the finite sensing period $T_{s}$ has not been removed. Only the MMoM method, which corrects and overcomes the impact of the finite sensing period $T_{s}$ on the estimation of the moments (in particular, the variance) can provide a nearly zero estimation error. These results demonstrate that only the proposed MMoM method can provide a nearly perfect estimation of the distribution of PU activity periods from spectrum sensing observations under realistic operation conditions.

Table I shows the KS distance as a function of the sensing period for the considered distribution estimation methods from simulations, versus the experimental KS distance from PECAS. The first point to note is that the experimental results match the ones obtained through simulations. The results shown in Table I prove that the proposed methods are accurate for $\mathrm{CR}$ systems. Moreover, these results have a significant importance for practical CR system design since they indicate that the proposed estimation methods allow an accurate estimation of primary traffic statics even with lowcost hardware devices as it the case of the PECAS prototype.

Next, the effect of sample size (i.e., number of idle/busy periods used to estimate the moments and subsequently the distribution) on the estimation accuracy of both MoM and MMoM methods is analysed. Fig. 6 shows the maximum KS distance for both methods versus the sample size for $T_{s}=0.2 t . u$. and an accurate knowledge of the minimum PU activity time (this is the case that provides the best possible accuracy). As it can be observed, the MMoM method requires a lower sample size. For example, for a target KS distance of $D_{K S}=0.1$, the MoM method requires the observation of 900 periods approximately while the MMoM method only requires around 300 period samples. Moreover, the MMoM estimation error decreases monotonically with the sample size, meaning that it can provide an arbitrarily accurate estimation of the distribution provided that a sufficiently large sample size is available, while the MoM method shows a lower bound below which it is impossible to reduce the estimation error no matter how large the sample size is.

\section{CONCLUSION}

The main focus of this work is to enable SU to obtain an accurate estimation of the distribution of PU activity periods, which plays a crucial role in improving the performance of $\mathrm{CR}$ systems and reducing the interference to primary networks. A modified version of the Method of Moments has been proposed to improve the primary distribution estimation. Simulation and experimental results have shown that the proposed method outperforms the conventional approach based on the direct estimation by means of empirical cdf calculation 
TABLE I

KS DISTANCE ESTIMATED USING PECAS (EXPERIMENT) VERSUS SIMULATION.

\begin{tabular}{|c|c|c|c|c|c|c|c|c|c|c|}
\hline \multirow{2}{*}{$\begin{array}{c}\mathbf{T}_{\mathbf{s}} \text { in } \\
\text { seconds }\end{array}$} & \multicolumn{9}{|c|}{ KS distance } \\
\cline { 2 - 12 } & Direct estimation & \multicolumn{1}{|c|}{ MoM with $\widehat{\mu}_{i}$} & \multicolumn{1}{c|}{ MoM with $\mu_{i}$} & \multicolumn{2}{c|}{ MMoM with $\widehat{\mu}_{i}$} & \multicolumn{2}{c|}{ MMoM with $\mu_{i}$} \\
\hline & Sim & Exp & Sim & Exp & Sim & Exp & Sim & Exp & \multicolumn{1}{|c|}{ Sim } & Exp \\
\hline 0.05 & 0.0431 & 0.0491 & 0.0095 & 0.0020 & 0.0081 & 0.0020 & 0.0094 & 0.0059 & 0.0038 & 0.0021 \\
\hline 0.10 & 0.0934 & 0.0947 & 0.0052 & 0.0018 & 0.0052 & 0.0018 & 0.0053 & 0.0021 & 0.0034 & 0.0021 \\
\hline 0.15 & 0.1086 & 0.1074 & 0.0439 & 0.0428 & 0.0288 & 0.0299 & 0.0431 & 0.0391 & 0.0036 & 0.0021 \\
\hline 0.20 & 0.1403 & 0.1399 & 0.0777 & 0.0723 & 0.0583 & 0.0572 & 0.0761 & 0.0706 & 0.0029 & 0.0022 \\
\hline 0.25 & 0.2079 & 0.2111 & 0.0051 & 0.0021 & 0.0045 & 0.0021 & 0.0042 & 0.0037 & 0.0030 & 0.0008 \\
\hline 0.30 & 0.1979 & 0.1961 & 0.1076 & 0.1055 & 0.1022 & 0.1018 & 0.1030 & 0.1009 & 0.0029 & 0.0024 \\
\hline 0.35 & 0.1825 & 0.1817 & 0.0446 & 0.0419 & 0.0789 & 0.0785 & 0.0462 & 0.0447 & 0.0024 & 0.0018 \\
\hline 0.40 & 0.1851 & 0.1840 & 0.0282 & 0.0270 & 0.0507 & 0.0500 & 0.0330 & 0.0319 & 0.0024 & 0.0015 \\
\hline 0.45 & 0.2714 & 0.2737 & 0.0127 & 0.0108 & 0.0239 & 0.0222 & 0.0159 & 0.0143 & 0.0023 & 0.0018 \\
\hline 0.50 & 0.3609 & 0.3626 & 0.0090 & 0.0079 & 0.0089 & 0.0079 & 0.0015 & 0.0010 & 0.0020 & 0.0010 \\
\hline
\end{tabular}

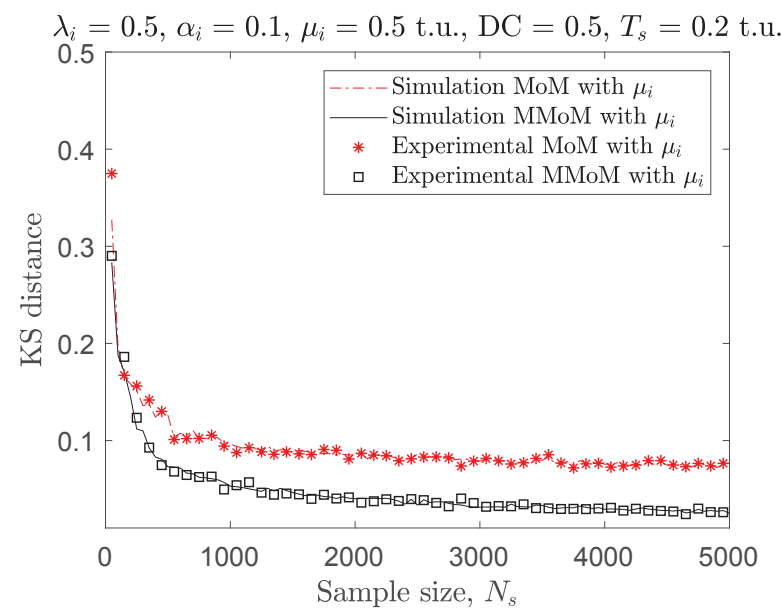

Fig. 6. KS distance for MoM and MMoM versus the sample size, both of the methods assumed with perfect $\mu_{1}$ knowledge.

as well as the approach based on the standard Method of Moments. It has been found out that an accurate estimation or knowledge of the minimum PU activity time is essential to achieve an accurate estimation of the distribution of PU activity periods. Provided that the minimum PU activity time is known or can be estimated to a sufficient degree of accuracy, the proposed MMoM method constitutes an ideal solution to provide an accurate (nearly perfect) estimation of the PU activity statistics based on spectrum sensing observations for practical applications.

\section{ACKNOWLEDGEMENTS}

M. López-Benítez would like to thank the support of British Council under UKIERI DST Thematic Partnerships 2016-17 (ref. DST-198/2017). K. Umebayashi would like to thank the support received from the MIC/SCOPE \#165003006, and JSPS KAKENHI Grant Numbers JP15K06053 and JP15KK0200.

\section{REFERENCES}

[1] S. Haykin, "Cognitive radio: Brain-empowered wireless communications," IEEE J. Sel. A. Comms., vol. 23, no. 2, pp. 201-220, Feb. 2005.

[2] M. López-Benítez and F. Casadevall, Spectrum Usage Models for the Analysis, Design and Simulation of Cognitive Radio Networks. Dordrecht: Springer Netherlands, 2012, pp. 27-73.
[3] M. Wellens, J. Riihijarvi, and P. Mahonen, "Empirical time and frequency domain models of spectrum use," Physical Communication, vol. 2, no. 1, pp. $10-32,2009$.

[4] X. Liu, B. Krishnamachari, and H. Liu, "Channel selection in multichannel opportunistic spectrum access networks with perfect sensing," in Proc. 2010 IEEE Int'l. Symp. Dyn. Spect. Access Networks (DySPAN 2010), Apr. 2010, pp. 1-8.

[5] C. H. Liu, W. Gabran, and D. Cabric, "Prediction of exponentially distributed primary user traffic for dynamic spectrum access," in 2012 IEEE Global Communications Conference, Dec 2012, pp. 1441-1446.

[6] T. Yucek and H. Arslan, "A survey of spectrum sensing algorithms for cognitive radio applications," IEEE Communications Surveys Tutorials, vol. 11, no. 1, pp. 116-130, First 2009.

[7] Q. Zhao, S. Geirhofer, L. Tong, and B. M. Sadler, "Opportunistic spectrum access via periodic channel sensing," IEEE Transactions on Signal Processing, vol. 56, no. 2, pp. 785-796, Feb 2008.

[8] K. Umebayashi, K. Moriwaki, R. Mizuchi, H. Iwata, S. Tiiro, J. J. Lehtomäki, M. López-Benítez, and Y. Suzuki, "Simple primary user signal area estimation for spectrum measurement," IEICE Transactions on Communications, vol. 99, no. 2, pp. 523-532, 2016.

[9] M. López-Benítez, "Can primary activity statistics in cognitive radio be estimated under imperfect spectrum sensing?" in IEEE Int'l. Symposium Pers., Indoor and Mobile Radio Comms. (PIMRC 2013), Sept 2013, pp. 750-755.

[10] C. H. Liu, P. Pawelczak, and D. Cabric, "Primary user traffic classification in dynamic spectrum access networks," IEEE J. Sel. Areas Comms. vol. 32, no. 11, pp. 2237-2251, November 2014.

[11] A. Al-Tahmeesschi, M. López-Benítez, K. Umebayashi, and J. Lehtomaki, "Analytical study on the estimation of primary activity distribution based on spectrum sensing," in IEEE Int'l. Symposium Pers., Indoor and Mobile Radio Comms. (PIMRC 2017), In Press.

[12] C. M. Grinstead and J. L. Snell, Introduction to probability. American Mathematical Soc., 2012.

[13] MATLAB, version 7.10.0 (R2016b). Natick, Massachusetts: The MathWorks Inc., 2016.

[14] M. López-Benítez and F. Casadevall, "Time-dimension models of spectrum usage for the analysis, design, and simulation of cognitive radio networks," IEEE Transactions on Vehicular Technology, vol. 62, no. 5 , pp. 2091-2104, Jun 2013.

[15] H. Kim and K. G. Shin, "Efficient discovery of spectrum opportunities with mac-layer sensing in cognitive radio networks," IEEE Transactions on Mobile Computing, vol. 7, no. 5, pp. 533-545, May 2008.

[16] N. Johnson, S. Kotz, and N. Balakrishnan, Continuous univariate distributions, ser. Wiley series in probability and mathematical statistics: Applied probability and statistics. Wiley \& Sons, 1995, no. v. 2.

[17] M. López-Benítez, "Cognitive radio," in Heterogeneous cellular networks: Theory, simulation and deployment. Cambridge University Press, 2013, ch. 13.

[18] M. López-Benítez, A. Al-Tahmeesschi, K. Umebayashi, and J. Lehtomaki, "PECAS: A low-cost prototype for the estimation of channel activity statistics in cognitive radio," in IEEE Wireless Comms. and Networking Conf. (WCNC 2017), March 2017, pp. 1-6.

[19] W. H. Press, S. A. Teukolsky, W. T. Vetterling, and B. P. Flannery, Numerical recipes: The art of scientific computing, $3 \mathrm{rd}$ ed. Cambridge University Press, 2007. 